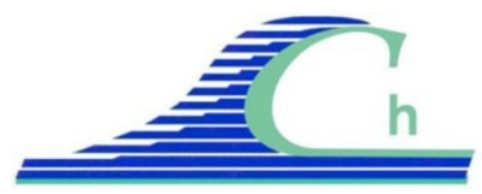

XII ${ }^{\text {ìmes }}$ Journées Nationales Génie Côtier - Génie Civil

Cherbourg, 12-14 juin 2012

DOI:10.5150/jngcgc.2012.110-D @ Editions Paralia CFL

disponible en ligne - http://www.paralia.fr - available online

\title{
État d'avancement d'un guide sur l'évaluation des risques sanitaires des opérations de dragage et de rejet en mer de matériaux marins et estuariens
}

\author{
Julie DROIT ${ }^{1}$, GEODE ${ }^{2}$
}

1. Cetmef, Direction de l'Ingénierie, Département Environnement et Aménagement, Division Environnement Aquatique et Énergies Renouvelables.

155 rue Pierre Bouguer, BP 5, 29280 Plouzané, France.

Julie.droit@developpement-durable.gouv.fr

2. Groupe d'Études et d'Observation des Dragages et de l'Environnement, Grand Port Maritime de Nantes Saint-Nazaire, 18 quai Ernest Renaud - BP 18609 - 44186 Nantes cedex 4, France.geode@nantes.port.fr

\section{Résumé :}

Dans le cadre des opérations de dragage et/ou de rejet en mer, un dossier d'étude d'impact est susceptible d'être réalisé. L'évaluation du risque sanitaire, qui est un des volets de ce dossier, permet d'appréhender les conséquences de ces travaux sur la santé des populations exposées.

De façon à harmoniser les pratiques, et à mettre à disposition des gestionnaires portuaires, des bureaux d'études, et des services instructeurs un référentiel commun, le Groupe d'Etudes et d'Observations des Dragages et de l'Environnement (GEODE) a décidé de produire un guide sur cette problématique.

Ce guide a pour objectif de proposer une méthode d'évaluation des risques sanitaires adaptée aux problématiques des opérations de dragage et d'immersion, et conforme aux directives et règlements européens.

La réalisation de ce guide est pilotée par le CETMEF, appuyé par l'ensemble des membres de GEODE, et un comité de suivi constitué de gestionnaires portuaires, de représentants de l'administration centrale et des services déconcentrés du Ministère de l'Écologie et du Ministère de la Santé, ainsi que de scientifiques d'Ifremer, de l'Ineris, et de l'Université de Rouen (microbiologie).

\section{Mots-clés :}

Dragage - Immersion - Rejet en mer - Risque sanitaire - Sédiments - Travaux maritimes - Environnement littoral - Étude d'impact - Port - Évaluation

\section{Introduction}

Le dragage est une opération qui permet de restaurer, entretenir, voire assainir des zones fortement impactées par l'accumulation de sédiments. Techniquement, les opérations de dragage correspondent à l'enlèvement ou au déplacement de sédiments à l'aide de moyens mécaniques, hydrauliques ou pneumatiques. 
Les rejets en mer correspondent, soit aux rejets effectués à l'aide de moyens nautiques ou via un émissaire, soit aux rejets directs des sédiments extraits au niveau de la drague (clapage).

Les opérations de dragage d'un montant supérieur à 1900000 euros, doivent faire l'objet d'un dossier d'étude d'impact (articles R.122-4, R.122-5 et R 122-8 du code de l'environnement). L'évaluation du risque sanitaire (ERS), qui est un des volets de ce dossier, permet d'appréhender les conséquences de ces travaux sur la santé des populations exposées.

Le cadre méthodologique proposé dans ce guide correspond à celui de la démarche d'évaluation des risques sanitaires, régie, en Europe, par la Directive 93/67/CEE et le règlement $\mathrm{CE} \mathrm{n}^{\circ} 1488 / 94$.

Ce guide méthodologique est destiné aux maîtres d'ouvrage, gestionnaires des ports, bureaux d'études, services instructeurs ou services techniques qui gèrent des opérations de dragage et de rejet en mer. Il doit leur permettre de disposer :

- d'éléments positionnant la problématique et de comprendre le contexte réglementaire encadrant l'évaluation des risques sanitaires ;

- d'une méthodologie d'évaluation des risques sanitaires des opérations de dragage et de rejet en mer de matériaux marins et estuariens ;

- d'éléments de réflexion opérationnelle et de gestion, à travers un ensemble de recommandations pratiques et d'exemples illustrant la méthodologie proposée.

\section{L'évaluation des risques sanitaires dans le cadre des opérations de dragage et d'immersion}

\subsection{La démarche générale de l'évaluation des risques sanitaires}

Afin de bien comprendre la démarche d'évaluation des risques sanitaires, nous nous attacherons dans un premier temps à définir les notions clés de cette démarche :

- L’impact sanitaire désigne les conséquences (éventuellement indirectes et/ou différées dans l'espace et dans le temps) d'un événement, d'un processus, d'une activité, ou d'une infrastructure, sur la santé.

- Le danger correspond aux caractéristiques intrinsèques des agents chimiques, physiques ou biologiques affectant la santé d'un individu.

- L'exposition correspond au contact entre un agent chimique, physique ou biologique qui présente des propriétés dangereuses et l’homme.

- Le risque correspond à la probabilité d'un événement considéré comme un mal ou un dommage.

- Le risque sanitaire correspond à la probabilité de survenue d'un événement (une maladie) pour un individu ou une population pendant une période donnée. Les risques à court terme, liés à une exposition aiguë, ne relevant pas d'une évaluation des 


\section{XII ${ }^{\text {èmes }}$ Journées Nationales Génie Côtier - Génie Civil \\ Cherbourg, 12-14 juin 2012}

risques sanitaires d'étude d'impact, seuls les risques sanitaires liés à une exposition chronique des populations aux substances à impact potentiel seront étudiés.

Selon la définition de l'U.S. National Research Council (NRC, 1983), la démarche d'évaluation des risques sanitaires consiste en "l'utilisation de faits scientifiques pour définir les effets sur la santé d'une exposition d'individus ou de populations à des matériaux ou à des situations dangereuses".

Elle comporte quatre étapes :

- L'identification du danger : quel effet néfaste ? Pour quelle voie d'exposition?

- Le choix de la valeur toxicologique de référence (VTR): ces valeurs reposent essentiellement sur des données toxicologiques qui permettent de définir des niveaux de toxicité au-delà desquels la probabilité d'observer des effets adverses est importante. Elles sont définies par différents organismes de recherche sur l'évaluation du risque sanitaire tels que IRIS (Integrated Risk Information System) de l'US-EPA (Environmental Protection Agency), l'ATSDR (Agency for Toxic Substances and Disease Registry), l'OMS (Organisation Mondiale de la Santé), Health Canada (Santé Canada), ou bien encore l'OEHHA (Office of Environmental Health Hazard Assessment).

- L'évaluation des expositions : quels sont les milieux contaminés ? Quelles sont les populations exposées ? Quelles sont les voies d'exposition (ingestion, inhalation, contact) ? Quels sont les durées et les niveaux d'exposition ?

- La caractérisation du risque: quelle probabilité de survenue du danger pour un individu dans une population donnée?

\subsection{L'évaluation des risques sanitaires appliquée aux opérations de dragage/immersion}

L’ERS nécessite un certain nombre de données permettant de définir le contexte du projet, les dangers potentiels, les voies d'exposition de la population, ainsi que l'identification des populations exposées.

Dans le contexte des opérations de dragage et de rejet en mer, ces données sont :

- Les cibles potentielles : zones conchylicoles, de pêche ou gisements coquillers naturels, zones de baignade, d'activités nautiques, etc ;

- La caractérisation chimique des sédiments de dragage, et la présence de rejets spécifiques, qui permettront d'identifier les contaminants potentiels ;

- Les caractéristiques des techniques utilisées et la caractérisation physique des sédiments, qui permettront de comprendre les phénomènes mis en jeu lors des opérations de dragage ou de rejet. En effet, selon la technique utilisée et la granulométrie des sédiments, la dispersion des sédiments sera différente ;

- La localisation des sites d'immersion ainsi que la connaissance du contexte hydrodynamique permettront d'identifier les zones les plus exposées.

Il apparaît clairement que ces informations ou données sont souvent identifiées lors de la mise en place d'un projet de dragage et de rejet. Par exemple, la caractérisation des 
sédiments est réalisée conformément à l'arrêté du 14 juin 2000 relatif aux niveaux de référence à prendre en compte lors de l'analyse des sédiments marins ou estuariens, et selon les niveaux GEODE établis pour certains contaminants (métaux, Polychlorobiphényles (PCB), tributylétain (TBT)).

Dans le cadre d'une ERS, ces niveaux ne serviront pas de référence puisqu'ils n'ont pas de rapport avec la santé humaine. Cependant, ces niveaux serviront de base pour évaluer les concentrations en contaminants auxquelles peut être exposée la population.

Ces concentrations pourront également être comparées à différentes valeurs réglementaires (normes de qualité des eaux de baignade, normes de qualité alimentaire, normes de qualité environnementale, ...), ou aux concentrations environnementales relevées dans les bases de données de différents organismes (Ifremer, Ineris, Agences de l'Eau, Cetmef), (LEVET et al., 2008 ; ALZIEU 1999 ; FURETOX, site web ; GIP SEINE AVAL, site web ; INERIS, site web ; CIRCA, site web).

A défaut de telles normes, les concentrations d'exposition pourront également être comparées à des valeurs toxicologiques de références (VTR), si elles existent.

\section{3. Établissement de critères décisionnels}

\section{1 Étude de faisabilité}

La méthodologie d'évaluation des risques sanitaires comporte une étape de "formulation du problème et de caractérisation du site" permettant notamment d'identifier les enjeux, les contaminants potentiels, et les scénarii d'exposition possibles.

Ces scénarii d'exposition doivent faire l'objet d'une analyse de leur pertinence à partir de critères décisionnels afin de ne retenir que ceux pour lesquels il n'est pas possible de conclure d'emblée en l'absence de risque sanitaire.

Pour les scénarii retenus, qui présenteraient un "doute", il sera alors possible de mettre en place des mesures compensatoires permettant de réduire les effets, de modifier certains aspects du projet, de réaliser des suivis environnementaux ou de mener une Évaluation Quantitative des Risques Sanitaires.

De façon à construire ces critères décisionnels, les données disponibles dans le domaine des opérations de dragage et d'immersion et de leur impact sur la santé humaine ont été inventoriées et analysées : dossiers réglementaires issus de différents ports français, publications scientifiques, entretiens avec des spécialistes, données extraites de sites internet spécialisés.

Le logigramme ci-après présente la méthodologie générale adoptée pour l'évaluation du risque sanitaire relative aux opérations de dragage et d'immersion.

D'après ce logigramme, nous avons identifié pour les opérations de dragage et de rejet en mer, les descripteurs déterminants et la possibilité de leur associer ou non des critères décisionnels :

- la dispersion des sédiments lors des opérations de dragage et d'immersion, 


\section{XII ${ }^{\text {èmes }}$ Journées Nationales Génie Côtier - Génie Civil \\ Cherbourg, 12-14 juin 2012}

- la contamination chimique des sédiments portuaires,

- la contamination biologique des sédiments portuaires.

L'étude de faisabilité concernant l'établissement des critères décisionnels a abouti aux conclusions suivantes :

- Dispersion des sédiments : des critères décisionnels ont été recherchés via l'analyse des données de distance entre les zones de travaux et les sites sensibles, d'orientation des courants et/ou des vents dominants, et de force des courants. Des valeurs majorantes de dispersion ont été trouvées dans la littérature. Cependant, compte tenu de la spécificité de chaque site, il a été conclu que ce type de critère ne pouvait pas être appliqué. Il est ainsi conseillé d'avoir recours à une modélisation spécifique à chaque site. En effet, la modélisation permet de rendre compte de la variabilité au niveau des différents sites et d'obtenir des distances non majorantes.

- Contamination chimique des sédiments: La seule voie d'exposition humaine à considérer est l'ingestion de produits de la mer (MARCHAND \& TISSIER, 2005). De façon à sélectionner les substances d'intérêt pour l'ERS, les données suivantes devront être recherchées :

- prise en compte par OSPAR et/ou par la Directive Cadre sur l'Eau (DCE) ;

- comparaison des caractéristiques des substances avec les critères de définition des substances PBT (persistantes, bioaccumulables et toxiques);

- classement dans la liste des produits CMR (cancérogène/mutagène/reprotoxique);

- importance de la prise en compte du critère sanitaire dans l'établissement des Normes de Qualité Environnementales (NQE) ;

- existence de VTR ou de seuils de consommabilité ;

- niveau de présence en milieu marin.

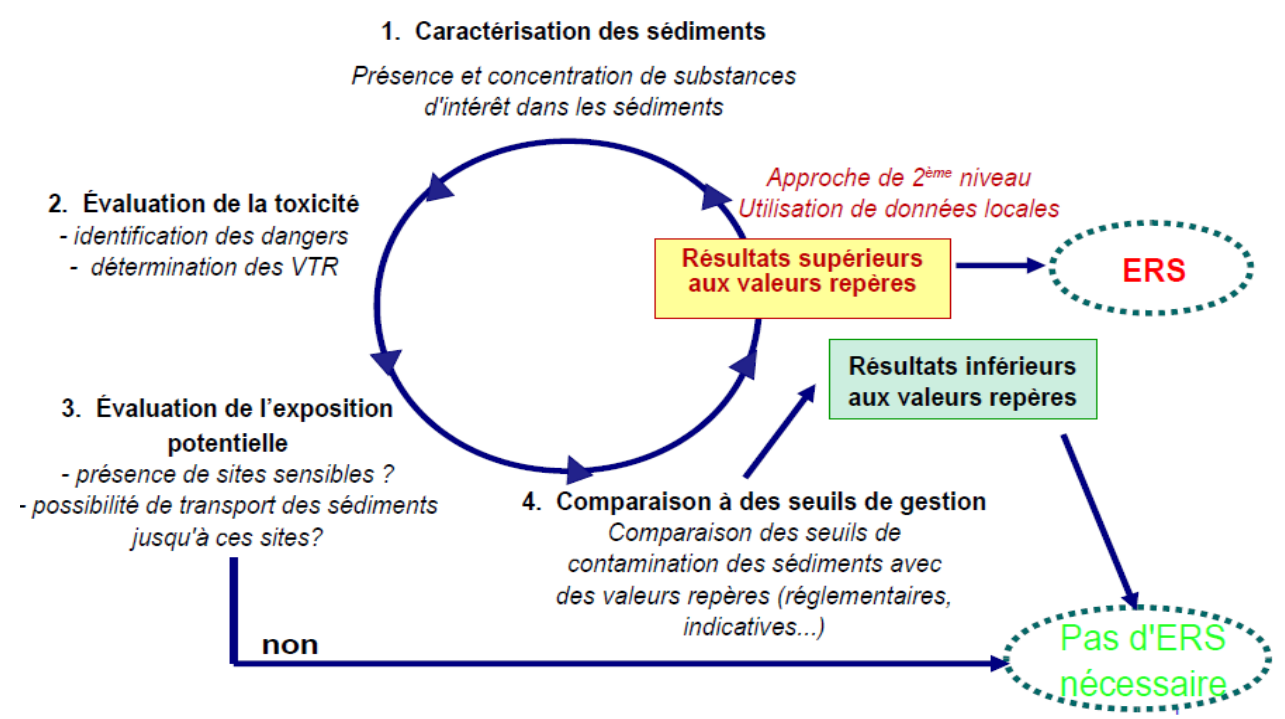

Figure 1. Schématisation de la démarche d'évaluation des risques adaptée à la problématique des dragages et de l'immersion. 
Pour ces substances, des valeurs repères de concentration dans les sédiments pourront être calculées via la méthode européenne d'équilibre de partage (Technical Guidance Document : TGD ; CE, 2003) à partir des seuils de consommabilité des produits de la mer, des VTR existantes, ou des Normes de Qualité Environnementales définies par la Directive Cadre sur l'Eau. En effet, cette méthode permet de passer des concentrations dans l'eau, aux concentrations dans le biote et les sédiments (voir MARCHAND \& TISSIER, 2005).

- Contamination biologique des sédiments : Les voies d'exposition pertinentes sont l'ingestion de produits de la mer, l'ingestion accidentelle d'eau de mer et le contact cutané.

- Bactéries, virus et parasites: Les critères décisionnels potentiels sont la contamination du substratum avant dragage, la granulométrie des sédiments dragués, la richesse en matière organique des sédiments, et la distance d'immersion ou de dragage par rapport aux sites sensibles.

- Phytoplancton toxique : Le seul critère décisionnel identifié est la situation du site de dragage en "zone à risque élevé" ou "zone à risque potentiel" définies selon les résultats du réseau de surveillance phytoplanctonique de l'Ifremer (REPHY).

Plusieurs études recommandent de rechercher dans les sédiments les kystes d'Alexandrium minutum. Si on se situe dans un secteur à risque, la détection de ces kystes pourrait donc être préconisée.

Le risque sanitaire sera ensuite dépendant de la proximité de sites sensibles (zones conchylicoles, de baignade, de loisirs nautiques...) et des possibilités de transfert des cellules phytoplanctoniques vers ces sites (distance, conditions hydrodynamiques, granulométrie des sédiments, ...).

3.2 Définition des critères décisionnels relatifs à la contamination chimique des sédiments

Les substances d'intérêt sanitaire ont été identifiées en fonction des critères précédemment cités et classés en 4 listes selon l'importance décroissante de leur prise en compte dans l'ERS (voir tableau 1):

- Pour les substances des listes 1 et 2 , des valeurs repères de concentration dans les sédiments ont été recherchées à partir des normes de qualité définies dans les produits de la mer, en utilisant la méthode européenne du TGD.

- Pour les substances des listes 3 et 4 qui font partie des substances prioritaires de la DCE, des "standards de qualité dans le biote" ont été calculés par la Commission Européenne (UE, 2005), sur la base de critères sanitaires. Des valeurs repères de concentration dans les sédiments ont donc pu être calculées via la méthode du TGD.

- Pour les autres substances (DDT, Aldrine, Dieldrine, Pyrène, Phénanthrène), la démarche européenne a été appliquée après recherche des VTR. 


\section{XII ${ }^{\text {èmes }}$ Journées Nationales Génie Côtier - Génie Civil \\ Cherbourg, 12-14 juin 2012}

Tableau 1. Listes des substances d'intérêt sanitaire dans les sédiments de dragage.

\begin{tabular}{|c|c|c|c|}
\hline Liste 1 & Liste 2 & Liste 3 & Liste 4 \\
\hline $\begin{array}{l}\text { Contaminants } \\
\text { réglementés concernant } \\
\text { leur teneur dans les } \\
\text { produits de la mer }\end{array}$ & $\begin{array}{l}\text { Contaminants faisant } \\
\text { l'objet de } \\
\text { recommandations } \\
\text { concernant leur teneur } \\
\text { dans les produits de la } \\
\text { mer }\end{array}$ & $\begin{array}{l}\text { Contaminants } \\
\text { prioritaires en milieu } \\
\text { marin et possédant une } \\
\text { VTR }\end{array}$ & $\begin{array}{l}\text { Contaminants pouvant } \\
\text { se retrouver en milieu } \\
\text { marin sur des bassins- } \\
\text { versants agricoles et } \\
\text { possédant une VTR }\end{array}$ \\
\hline $\begin{array}{l}\text { Arsenic } \\
\text { Benzo(a)pyrène } \\
\text { Cadmium } \\
\text { Plomb } \\
\text { Mercure } \\
\text { PCB-DL,dioxines, et } \\
\text { furanes } \\
\text { Hexachlorobenzène } \\
\text { (NQEbiote) }\end{array}$ & $\begin{array}{l}\text { PCB indicateurs (CB 28, } \\
\text { 52, 101, 118, 138, 153, 180) } \\
\text { Anthracène } \\
\text { Fluoranthène } \\
\text { Naphtalène } \\
\text { Benzo(b)fluoranthène } \\
\text { Benzo(ghi)pérylène } \\
\text { Benzo(k)fluoranthène } \\
\text { Benzo(a)anthracène } \\
\text { Indéno(1,2,3-cd)pyrène } \\
\text { Chrysène }\end{array}$ & $\begin{array}{l}\text { Pyrène } \\
\text { Phénanthrène } \\
\text { Nickel } \\
\text { DEHP } \\
\text { Lindane } \\
\text { Nonylphénols } \\
\text { Tributylétain (TBT) } \\
\text { DDT }\end{array}$ & $\begin{array}{l}\text { Chlorpyrifos } \\
\text { Endosulfan } \\
\text { Aldrine } \\
\text { Dieldrine }\end{array}$ \\
\hline
\end{tabular}

Parmi ces substances, les éléments métalliques, les PCB indicateurs, le TBT et les HAP sont suivis en routine lors des analyses des sédiments à draguer.

La majorité des autres substances sont suivies dans le cadre du Réseau national de surveillance de la qualité de l'eau et des sédiments dans les ports maritimes (REPOM) mis en place en 1997. En effet, depuis 2010, et pour une période de 3 ans, le programme eau est suspendu, tandis que le programme sédiment est complété par certaines substances prioritaires DCE et OSPAR.

Seuls le Chlorpyrifos, les PCB-DL, les dioxines et les furanes ne font pas l'objet d'un suivi en routine.

3.3 Définition des critères décisionnels relatifs à la contamination microbiologique des sédiments

La définition de critères décisionnels concernant la contamination microbiologique des sédiments se heurte à un manque d'informations précises concernant :

- les temps de survie des agents pathogènes en fonction du type de sédiment : il manque des données concernant l'association des agents pathogènes en fonction de la granulométrie et des caractéristiques physico-chimiques des sédiments ;

- les indicateurs bactériens pouvant être utilisés en milieu marin : certaines études mettent en avant un manque de corrélation entre E. coli (indicateur de contamination fécale retenu par les normes européennes), et certains agents pathogènes (notamment les virus) pouvant être plus résistants en milieu marin. La possibilité de suivre d'autres germes indicateurs ou directement des germes pathogènes doit donc être étudiée.

De façon à compléter le guide sur cet aspect, une expertise est en cours. 


\section{Conclusions et perspectives}

Des valeurs repères de concentrations dans les sédiments ont été calculées selon la méthode décrite précédemment.

Les valeurs repères qui paraissent cohérentes vis à vis des niveaux de concentration rapportés dans la littérature sont actuellement discutées au sein de GEODE, et n'ont pas encore été validées en tant que critères décisionnels.

Une expertise scientifique doit être engagée pour ce qui concerne l'établissement de critères décisionnels relatifs à la contamination microbiologique des sédiments : sélection des micro-organismes à rechercher dans les cas à risque, recherche de corrélations entre type de sédiment et survie des micro-organismes en milieu marin..

Lorsque les critères décisionnels auront été établis, la suite de l'étude consistera à adapter la méthodologie d'évaluation des risques sanitaires définie par l'Europe, au cas des opérations de dragage et d'immersion, et à tester cette méthodologie sur deux cas concrets.

Le guide final sera ensuite diffusé aux gestionnaires portuaires, aux services instructeurs, et aux bureaux d'études travaillant sur ces problématiques.

\section{Références}

AESN -Agence de l'Eau Seine Normandie- (2008). Guide pratique des substances toxiques dans les eaux douces et littorales du bassin Seine-Normandie. 271 p.

ALZIEU C. (1999). Dragage et environnement marin. Etat des connaissances. Ed. Ifremer $223 \mathrm{p}$.

CE (2003). Technical guidance document (TGD) on risk assessment of chemical substances following European regulations and directive. $2^{\text {nd }}$ édition.

CIRCA (site web). http://circa.europa.eu

FURETOX (site web). http://www.furetox.fr/

GIP SEINE AVAL (site web). http://seine-aval.crihan.fr/web/

INERIS (site web). Fiches de données toxicologiques et environnementales des substances chimiques : http://www.ineris.fr/substances/fr/page/21

LEVET D., LE HEN A, JACQUES S., MOUCHELIN J., BERLY A. (2008). Guide pratique des substances toxiques dans les eaux douces et littorales du bassin SeineNormandie. Rapport AQUASCOP pour l'Agence de l'Eau Seine Normandie, 271 p.

MARCHAND M., TISSIER C. (2005). Analyse du risque chimique en milieu marin. L'approche méthodologique européenne.. Ed. Ifremer, 126 p.

NRC -National Research Council- (1983). Risk assessment in the federal government: managing the process. National academy press, Washington D.C., USA, 191 p.

UE (2005). Environmental Quality Standards (EQS), Substance data sheet for priority substance. Disponible en ligne sur http://circa.europa.eu/ 